\begin{tabular}{cc}
\hline & International Journal of Engineering \& Technology, $7(3.6)(2018) 267-269$ \\
SPC & International Journal of Engineering \& Technology \\
\hline
\end{tabular}

\title{
Exact Solution for Unsteady Flow of Viscous Incompressible Fluid Over a Suddenly Accelerated Flat Plate (Stokes' First Problem) Using Laplace Transforms
}

\author{
Spainborlang Kharchandy ${ }^{1}$ \\ ${ }^{1}$ Department Of Mathematics, Shillong College, Shillong, India.
}

\begin{abstract}
With the Navier-Stokes Equation in Cartesian form (in absence of body forces), Laplace Transforms provides a simple approach towards solving the unsteady flow of a viscous incompressible fluid over a suddenly accelerated flat plate. On comparing the results between Laplace Transforms and similarity methods, it reveals that Laplace Transforms is simple and effective.
\end{abstract}

\section{Introduction}

The problem of unsteady flow of viscous incompressible fluid over a Suddenly accelerated flat plate was first solved by G.G.Stokes in the year 1856 in his famous treatment of the pendulum. Later in the year 1911, Lord Rayleigh also treated this flow and it often called the Rayleigh problem in the Literature. In many cases, this Stokes' First Problem is also known as 'the startup' flow problem i.e problem related with motion from rest.

The Laplace Transform or Laplace Transformation is a method for solving many differential operations arising in Physics and Engineering. By the way, the Laplace Transformation is just one of many Integral transform in general use. Conceptually and computationally, it is probably the simplest. Indeed, if one can understand the Laplace Transform, then he or she will find it much easier to pick up other transformations as needed.

The Laplace Transform is defined by

$F(p)=L\{f(t)\}=\int_{0}^{\infty} e^{-p t} f(t) d t \quad ; \quad t \geq 0$

\section{Some Basic Terminologies}

1. Unsteady flow: The flow is said to be unsteady when the conditions and fluid property $(P$,say) at any point of the flow field change with regard to the time. For example, Water being pumped through a fixed system at an increasing rate represents Unsteady flow.

$$
\text { Mathematically, } \quad \frac{\partial P}{\partial t} \neq 0
$$

2. 2. Viscosity: Each element of a fluid experiences stress exerted on it by other elements of the fluid which surround it. The stress at each part of the surface of the element is resolved into two components: Normal and Tangential to the surface( see figure 1.1), known as pressure and shearing stress respectively. Pressure are exerted whether the fluid is moving or at rest, but shear stress occurs only in moving fluids. This feature is the one which enables fluid to distinguished from solids. The property which give rise to shear stress is called Viscosity. Viscosity arise when there is a relative motion between different fluid layers. It possessed by all real fluids.

Viscous fluid is defined as the fluid where normal as well as shearing stresses exist .For example, Syrup and Heavy oil are treated as viscous fluids.

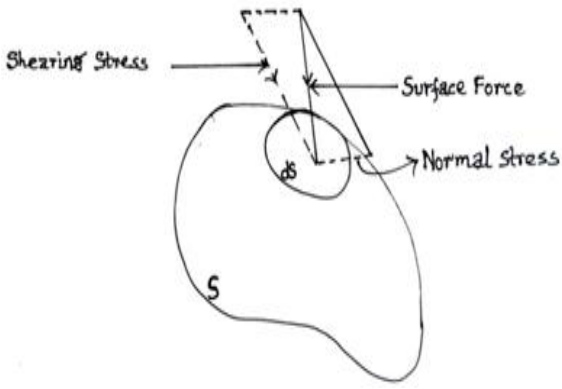

Figure 1.1

3. Incompressible fluid : The compressibility of a fluid is defined as the variation of its density, with the variation of pressure. For example, LPG, Nitrogen gas, etc.

Therefore, a fluid is said to be incompressible if it requires a large variation in pressure to produce some variation in density. For example, water, air(to some extent), etc.

4. Newtonian fluids: The fluids in which the stress components (Shearing stress, $\tau$ ) are linear functions of rate of strain components (rate of deformation, $\left(\frac{\partial u}{\partial y}\right)$ ) are termed as Newtonian fluids. For example, Real fluids and Ideal fluids.

Mathematically, $\quad \tau=\mu\left(\frac{\partial u}{\partial y}\right)$

[ $\mu$ : the constant of proportionality (or, Coefficient of viscosity or, Dynamic Viscosity or, Viscosity]

\section{Stokes' First Problem(Start-Up Flow Problem)}

Stokes' first problem is a fundamental solution in fluid dynamics which represents one of the few exact solution to the Navier- 
Stokes equations. Consider a Cartesian coordinate system of an infinite long flat plate extending to a large distances in the $X$ and $Z$-directions. Let there be an incompressible viscous fluid occupying the half plane $y=0$ (i.e $x z-$ plane). At $t=0$, the plate is suddenly set in motion at a constant velocity $U_{0}$ in the $X$-direction.

This generates a one-dimensional parallel flow near the plate as shown in figure 1.2

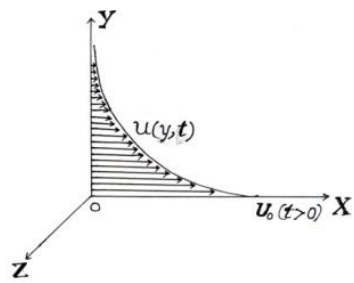

Figure 1.2

$$
\text { i.e } \quad v=0, w=0, \quad \frac{\partial}{\partial z}=0
$$

(i.e the only non-zero velocity component will be $u$ which will be a function of $y \& t$ )

The Continuity Equation reduces to

$\frac{\partial u}{\partial x}=0, \quad$ so that $u=u(y, t)$

Since the plate is situated in an infinite fluid, the Pressure must be constant everywhere (i.e. Pressure (, say) is independent of $x \Rightarrow$ $\left.\frac{\partial P}{\partial x}=0\right)$

Hence, the Navier-Stokes equations in absence of body forces reduces to

$\frac{\partial u}{\partial t}=v \frac{\partial^{2} u}{\partial y^{2}}$

Which is to be solved under the following Initial condition and Boundary Conditions of the problem:

$$
\begin{aligned}
& u=0 \text { when } t \leq 0 \text { for all } y \\
& \left.\begin{array}{cc}
u=U_{0} & \text { at } y=0 \\
u=0 & \text { at } y=\infty
\end{array}\right\} \quad \text { when } t>0
\end{aligned}
$$

As we have the governing equation, Initial condition and Boundary conditions ; therefore, the problem is well-posed. We utilize the Laplace Transform method to reduce the two variables into a single variable i.e transferring partial differential equation into ordinary differential equation.

On using Laplace Transform technique, equation (4) and the Boundary Conditions takes the following form:

$$
\left.\begin{array}{c}
\begin{array}{c}
d^{2} \widetilde{u}(y, p) \\
d y^{2}
\end{array}-\frac{p}{v} \widetilde{u}(y, p)=0 \\
\widetilde{u}(0, p)=\frac{U_{0}}{p}, \widetilde{u}(\infty, p)=0
\end{array}\right\}
$$

Where $\quad L\left\{\frac{\partial u}{\partial y}\right\}=\frac{d \tilde{u}}{d y} \Rightarrow L\left\{\frac{\partial^{2} u}{\partial y^{2}}\right\}=\frac{d^{2} \widetilde{u}}{d y L^{2}}$

and

$L\left\{\frac{\partial u}{\partial t}\right\}=L\left\{u^{\prime}(t)\right\}=\int_{0}^{\infty} e^{-p t} u^{\prime}(t) d t=\left[e^{-p t} u(t)\right]_{0}^{\infty}-\int_{0}^{\infty}(-p) e^{-p t} u(t) d t$ (on Integrating by parts)

$$
\text { or, } \begin{aligned}
L\left\{\frac{\partial u}{\partial t}\right\}= & 0-u(y, 0)+p \int_{0}^{\infty} e^{-p t} u(t) d t \\
& =p L\{u(t)\}-u(y, 0) \\
& =p \tilde{u}(y, p)-0=p \tilde{u}(y, p)
\end{aligned}
$$

The general equation of (6) is

$$
\tilde{u}(y, p)=A e^{\sqrt{\frac{p}{v}} y}+B e^{-\sqrt{\frac{p}{v}} y}
$$

Since $u(y, t)$ must be bounded as $y \rightarrow \infty$, we must have $\tilde{u}(y, p)$ also bounded as $y \rightarrow \infty$. It follows that we must choose $A=0$ provided $\sqrt{p}>0$.

Therefore (7) reduces to $\tilde{u}(y, p)=B e^{-\sqrt{\frac{p}{v}} y}$

Subjecting to boundary conditions, we have

$$
\tilde{u}(0, p)=B \Rightarrow \frac{U_{0}}{p}=B
$$

So, equation (8) takes the form

$$
\tilde{u}(y, p)=\frac{U_{0}}{p} e^{-\sqrt{\frac{p}{v}} y}
$$

On taking inverse Laplace Transform in (9), the velocity profile $u(y, t)$ is given by

$$
\begin{gathered}
L^{-1}\{\widetilde{u}(y, p)\}=L^{-1}\left\{\frac{U_{0}}{p} e^{-\sqrt{\frac{p}{v}} y}\right\} \\
\Rightarrow u(y, t)=U_{0} L^{-1}\left\{\frac{e^{-\sqrt{\frac{p}{v}} y}}{p}\right\}
\end{gathered}
$$

Therefore,

$u(y, t)=U_{0} \operatorname{erfc}\left(\frac{y}{2 \sqrt{v t}}\right)=U_{0} \operatorname{erfc}(\eta)$

or, $\quad u(y, t)=U_{0}(1-\operatorname{erf}(\eta))$

(where $\eta=\frac{y}{2 \sqrt{v t}}$ )

Equation (10) can be written as :

$u(y, t)=U_{0}\left[1-\frac{2}{\sqrt{\pi}} \int_{0}^{\eta} e^{-l^{2}} d l\right]$

On using General Leibnitz 's Rule ,

We have from (11)

$\frac{\partial u(y, t)}{\partial y}=\frac{\partial}{\partial y}\left[U_{0}-\frac{2 U_{0}}{\sqrt{\pi}} \int_{o}^{\eta} e^{-l^{2}} d l\right]$

or, $\frac{\partial u}{\partial y}=\frac{\partial U_{0}}{\partial y}-\frac{2 U_{0}}{\sqrt{\pi}}\left[\int_{0}^{\frac{y}{2 \sqrt{v t}}} \frac{\partial\left(e^{-l^{2}}\right)}{\partial y} d l+\frac{1}{2 \sqrt{v t}} \cdot e^{-\frac{y^{2}}{4 v t}}-e^{-\frac{y^{2}}{4 v t}} \cdot 0\right]$ (on replacing $\eta$ by $\frac{y}{2 \sqrt{v t}}$ )

or, $\frac{\partial u}{\partial y}=0-\frac{2 U_{0}}{\sqrt{\pi}}\left[\int_{0}^{\frac{y}{2 \sqrt{v t}}}(-2 l) e^{-l^{2}} d l+\frac{1}{2 \sqrt{v t}} \cdot e^{-\frac{y^{2}}{4 v t}}\right]$ or, $\frac{\partial u}{\partial y}=-\frac{2 U_{0}}{\sqrt{\pi}}\left[e^{-\frac{y^{2}}{4 v t}}-1+\frac{e^{-y^{2}}}{2 \sqrt{v t}}\right]$

Therefore

$\left(\frac{\partial u}{\partial y}\right)_{y=0}=-\frac{2 U_{0}}{\sqrt{\pi}}\left[1-1+\frac{1}{2 \sqrt{v t}}\right]$

Or, $\left(\frac{\partial u}{\partial y}\right)_{y=0}=-\frac{U_{0}}{\sqrt{\pi v t}}$

Finally, it is of interest to determine the drag at the plate. Therefore the Skin friction (the Shearing Stress at the plate) is given by $\tau_{\omega}$ where

$$
\tau_{\omega}=\mu\left(\frac{\partial u}{\partial y}\right)_{y=0}=\mu\left(-\frac{U_{0}}{\sqrt{\pi v t}}\right)=-\frac{\rho v U_{0}}{\sqrt{\pi v t}} \quad(\text { using (12)) }
$$

so, $\quad \tau_{\omega}=-\rho U_{0} \sqrt{\frac{v}{\pi t}}$

From (13), We see that at initial point (i. e at $t=0$ ), the wall Shear Stress is infinite and it decreases to Zero in proportion to $\frac{1}{\sqrt{t}}$. It is also proportional to $\sqrt{v}$. It means here that a large force is needed to set the Fluid in motion.

and Coefficient of Skin friction $=\frac{\tau_{\omega}}{\frac{1}{2} \rho U_{0}^{2}} \quad=$ $-\frac{2 \rho U_{0}}{\rho U_{0}^{2}} \cdot \sqrt{\frac{v}{\pi t}}=-\frac{2}{U_{0}} \sqrt{\frac{\nu}{\pi t}}$.

\section{Conclusion}

In this connection, we successfully applied Laplace Transform to solve the Stokes' first problem (A Newtonian Fluid Problem). The result is identical to the one given in Literature. It gives a simple 
and a powerful mathematical tool. This Result reveals that the method is simple and effective.

\section{References}

[1] Schlichting H \& Gersten K, Boundary Layer Theory (Springer), $8^{\text {th }}$ Edition, (2016).

[2] Kleinstreuer C, Engineering Fluid Dynamics (An Interdisciplinary Systems Approach, Cambridge University Press, (1997).

[3] Achenson DJ, Elementary Fluid Dynamics, Oxford University Press, (2009), pp.26-40.

[4] Raisinghania MD, Fluid Dynamics, S.Chand and Company Ltd., (2005), pp.717-720.

[5] Swarup S, Fluid Dynamics, Krishna Prakashan Media(P) Ltd, (2009), pp.570-572

[6] Raisinghania MD, Saxena $\mathrm{HC}$ \& Dass HK, Integral Transforms(Laplace \& Fourier Transforms), S.Chand and Company Ltd., (2003).

[7] Vasishtha AR \& Gupta RK, Integral Transforms, Krishna Prakashan Media(P) Ltd. ,Meerut, (2008). 